\title{
Matching Dry to Wet Materials
}

\author{
Yaser Yacoob \\ Computer Vision Laboratory \\ University of Maryland, College Park \\ yaser@umiacs. umd. edu
}

\begin{abstract}
When a translucent liquid is spilled over a rough surface it causes a significant change in the visual appearance of the surface. This wetting phenomenon is easily detected by humans, and an early model was devised by the physicist Andres Jonas Angstrom nearly a century ago. In this paper we investigate the problem of determining if a wet/dry relationship between two image patches explains the differences in their visual appearance. Water tends to be the typical liquid involved and therefore it is the main objective. At the same time, we consider the general problem where the liquid has some of the characteristics of water (i.e., a similar refractive index), but has an unknown spectral absorption profile (e.g., coffee, tea, wine, etc.). We report on several experiments using our own images, a publicly available dataset, and images downloaded from the web.
\end{abstract}

\section{Background}

When a material absorbs a liquid it changes visual appearance due to richer light reflection and refraction processes. Humans easily detect wet versus dry surfaces, and are capable of integrating this ability in object detection and segmentation. As a result, a wet part of a surface is associated with the dry part of the same surface despite significant differences in their appearance. For example, when driving over a partially wet road surface it is easily recognized as a drivable surface. Similarly, a wine spill on a couch is recognized as a stain and not a separate object. The same capability is harder to implement in computer vision since the basic attributes of edges, color distributions and texture are disrupted in the wetting process. Engineering algorithms around these changes has not received attention in published research. Nevertheless, such capability is needed to cope with partial wetting of surfaces.

The emphasis of this paper is on surfaces combining both

This work was partially supported by the Office of Naval Research under Grant N00014-10-1-0934.
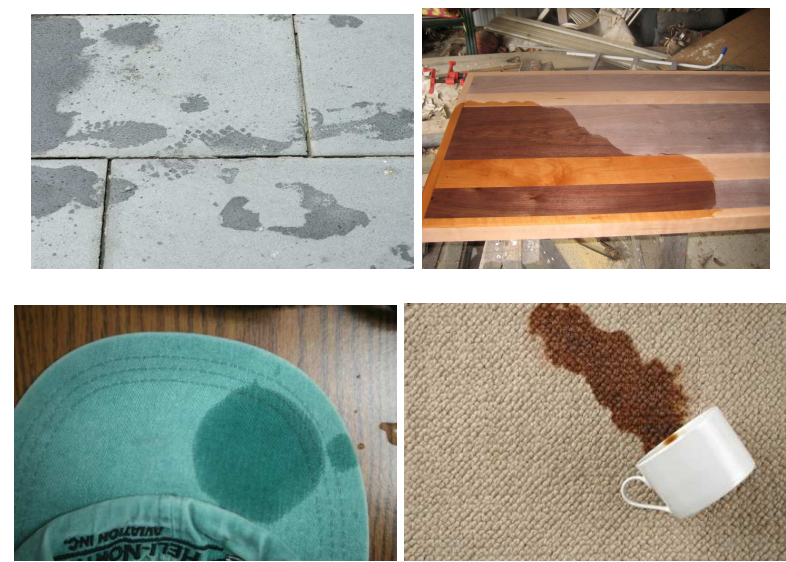

Figure 1. A partially wet concrete pavement, water spilled on wood, water stain on a cap, and coffee spilled on a carpet.

dry and wet parts. Distinguishing between completely wet and dry surfaces in independent images requires accounting for the illumination variations in the scenes, and may be subject to increased ambiguity in the absence of context. For example, comparing an image of a dry T-shirt to an image of the same T-shirt taken out of a washing machine is a more challenging problem since the straightforward solution is to consider them as different colored T-shirts. However, the algorithms we develop in this paper apply to this scenario assuming illumination is the same in both images.

Figure 1 shows examples we analyze: (a) partially wet concrete pavement, (b) water spilled on a piece of wood, (c) water stain on a cap, and (d) coffee spilled on a carpet. We assume that the wet and dry patches have been pre-segmented and focus on whether the dry patch can be synthesized to appear wet under unknown parameters employing a well-known optical model.

There are several factors that determine the visual appearance of wet versus dry surfaces. Specifically:

- The physical properties of the liquid involved. The translucence (or light absorption) of the liquid determines if interreflection occurs and is visually observed. Water is translucent, while paint is near opaque. The light absorption of the liquid as a function of wave- 
lengths affects the overall spectral appearance of the wet area. Water absorbs slightly more of the green and red wavelengths and less of the blue wavelength, while olive oil absorbs more of the blue wavelength and much less of the red and green wavelengths.

- The size and shape of the liquid affect the optical properties of the scene. For example, liquid droplets create a complex optical phenomenon as the curvature of each droplet acts as a lens (e.g., a drop of water can operate as a magnifying lens as well as cause light dispersion).

- The illuminant contributes to the appearance of both the dry and wet patches since it determines the wavelengths that are reaching the scene and the absorptions of the surface and liquid.

- The liquid absorption rate of the material determines whether a thin film of liquid remains floating apart on top of the material surface. For example, some plastics or highly polished metals absorb very little liquid and therefore a wetting phenomenon without absorption occurs. Nevertheless, non-absorbed liquids do change the appearance of the surface as they form droplets.

- Specular reflections may occur at parts of the wet surface and therefore mask the light refraction from air-toliquid and interreflections that occur within the liquidmaterial complex.

In this paper we study the problem of determining if two patches within the same image (or two images taken under similar illumination conditions) can be explained as wet and dry instances of the same material given that the material, liquid and illumination are unknown.

The paper's contribution is proposing an algorithm for searching a high-dimensional space of possible liquids, material and imaging parameters to determine a plausible wetting process that explains the appearance differences between two patches. Beyond the basic aspects of the problem, the results are relevant to fundamental capabilities such as detection, segmentation and recognition.

\section{Related Research}

Wet surfaces were considered first as an optics albedo measurement of various surfaces by Angstrom in 1925 [1]. The proposed model assumed that light reaching the observer is solely stemming from rays at or exceeding the critical angle and thus the model suggested less light than experimental data. Lekner and Dorf [3] expanded this model by accounting for the probability of internal reflections in the water film and the effect of the decrease of the relative refractive index at the liquid to material surface. Ther model was shown to agree more closely with experimental data.
In computer graphics, Jensen et al. [5] rendered wet surfaces by combining a reflection model for surface water with subsurface scattering. Gu et al [6] observed empirically the process of surface drying of several materials but no physical model for drying was offered. There has been little interest in wet surfaces in computer vision. Mall and da Vitoria Lobo [4] adopted the Lekner and Dorf model [3] to convert a dry material into a wet appearance and vice versa. The algorithm was described for greyscale images and fixed physical parameters. This work forms the basis of our paper. Teshima and Saito [2] developed a temporal approach for detection of wet road surfaces based on the occurrence of specular reflections across multiple images.

\section{Approach}

Given two patches, $P_{d}$ presumed dry, and $P_{w}$ possibly wet, the objective is to determine if a liquid of unknown properties can synthesize the dry patch so that it appears visually similar to the wet patch. We employ the term material to describe the surface that absorbs the thin film of liquid to create the wet patch. We leverage the optical model developed by [3] and used by [4], by formulating a search over the parameter space of possible materials and liquids.

In this paper we focus on a partial set of liquid on material appearances. Specifically, we exclude specular reflections, non-absorbing materials, and liquid droplets.

\subsection{Optics Model}

Figure 2 shows the basic model developed in [3]. A light ray entering the liquid film over the rough material surface with a probability of $1-R_{l}$ where $R_{l}$ is the reflectance at the air-liquid interface. A fraction, $a$, of this light is absorbed by the material surface, and thus $\left(1-R_{l}\right) *(1-a)$ is reflected back to the liquid surface. Let $p$ be the fraction of light reflected back into the liquid at the liquid-air surface. The total probability of absorption by the rough surface as this process repeats is described by

$$
A=\left(1-R_{l}\right)\left[a+a(1-a) p+a(1-a)^{2} p^{2}+\ldots\right]=\frac{\left(1-R_{l}\right) a}{1-p(1-a)} .
$$

Lekner and Dorf [3] show that $p$ can be written in terms of the liquid 's refractive index $n_{l}$ and the average isotropically illuminated surface $\bar{R}$ :

$$
p=1-\frac{1}{n_{l}^{2}}\left[1-\bar{R}\left(n_{l}\right)\right]
$$

where $\bar{R}(n)(n>1)$ :

$$
\begin{array}{r}
\bar{R}(n)=\frac{3 n^{2}+2 n+1}{3(n+1)^{2}}-\frac{2 n^{3}\left(n^{2}+2 n-1\right)}{\left(n^{2}+1\right)^{2}\left(n^{2}-1\right)}+\frac{n^{2}\left(n^{2}+1\right)}{\left(n^{2}-1\right)^{2}} \log (n) \\
-\frac{n^{2}\left(n^{2}-1\right)^{2}}{\left(n^{2}+1\right)^{3}} \log \left(\frac{n(n+1)}{n-1}\right)
\end{array}
$$




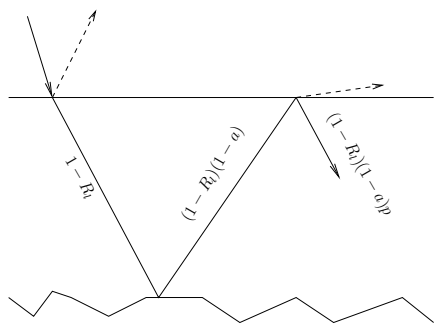

Figure 2. The light air-to-liquid and liquid-to-surface model.

Lekner and Dorff [3] proposed that the light absorption rates of the dry and wet materials are different, and that the wet material will always have a higher absorption rate. Let $a_{d}$ and $a_{w}$ be the light absorption rates of the dry and wet materials respectively, so that $a_{w}>a_{d}$. Thus the albedo values for the dry and wet surfaces are $1-a_{d}$ and $A=1-$ $a_{w}$, respectively, assuming isotropic illumination. Let $n_{r}$ be the refractive index of the material. For small absorptions, $a_{d} \approx 1-\bar{R}\left(n_{r}\right)$, and $a_{w} \approx 1-\bar{R}\left(n_{r} / n_{l}\right)$ and therefore

$$
a_{w} \approx a_{d}\left[1-\bar{R}\left(n_{r} / n_{l}\right)\right] /\left[1-\bar{R}\left(n_{r}\right)\right]
$$

while for large absorptions $a_{w} \approx a_{d}$. An interpolation of the two values can be expressed as

$$
a_{w}=a_{d}\left(1-a_{d}\right) \frac{1-\bar{R}\left(n_{r} / n_{l}\right)}{1-\bar{R}\left(n_{r}\right)}+a_{d}
$$

\subsection{Imaging Model}

Lekner and Dorff [3] and Mall and da Vitoria Lobo [4] focused on the albedo change between dry and wet surfaces. The model is suitable for estimating reflectance of a single wavelength but requires extension to aggregated wavelengths captured by greyscale or color images. In [4], the model was applied to greyscale images where the true albedo was approximated by using the maximum observed brightness in the patch. This assumes that micro-facet orientations of the material are widely distributed. Color images present two additional issues: cameras (1) integrate light across spectral zones, and (2) apply image processing, enhancement and compression to the raw images. As a result, the input image is a function of the actual physical process but may not be quantitatively accurate.

Our objective is to estimate the albedo of the homogeneous dry patch, $P_{d}$, for each of the $R G B$ channels (overlooking the real spectral wavelengths), despite unknown imaging parameters. It is critical to note that the camera acquires an image that is a function of the albedo, surface normal and illuminant attributes (direction, intensity and emitted wavelengths) at each pixel, so that estimating the true physical albedo is challenging in the absence of information about the scene. In the following we first describe a representation of the relative albedo in $R G B$ and then describe how it is re-formulated to derive possible absolute albedo values.
Let the albedo of the homogeneous dry material be $A_{R}, A_{G}, A_{B}$ with respect to the RGB channels. Then,

$$
A_{R}=1-a_{R}, A_{G}=1-a_{G}, A_{B}=1-a_{B}
$$

where $a_{R}, a_{G}, a_{B}$ are the absorption rates of light in the red, green and blue channels, respectively. Since the value of each absorption parameter is between 0 and 1 , it is possible to search this three dimensional space in small increments of $a_{R}, a_{G}, a_{B}$ values. However, these absorption rates are confounded with the variable surface normals across the patch as we consider $R G B$ values. Instead, we observe that the colors of pixels reflect, approximately, the relative absorption rates of red, green and blue. For example, a grey pixel indicates equal absorption in red, green and blue regardless of the level of the greyness. The surface normal contributes to a scalar that modifies the amount of light captured by the camera, but does not alter the relative albedos. Therefore, we can parametrize the albedo values as $A_{R} *\left(1, r_{G R}, r_{B R}\right)$, where $r_{G R}$ and $r_{B R}$ are the relative albedo values green-to-red and blue-to-red, respectively. This parametrization does not, theoretically, change due to variation in surface normals. Specifically, consider a homogeneous patch of constant albedo but variable surface normals, and assuming a Lambertian model, the image reflectance can be expressed as

$$
\begin{aligned}
& I_{R}(x, y)=A_{R} *(N(x, y) \cdot S(x, y)) \\
& I_{G}(x, y)=A_{G} *(N(x, y) \cdot S(x, y)) \\
& I_{B}(x, y)=A_{B} *(N(x, y) \cdot S(x, y))
\end{aligned}
$$

where $N(x, y)$ and $S(x, y)$ are the surface normal and the illuminant direction at $(x, y)$, respectively $(S(x, y)=S$ for a distant point light source). The two ratios $r_{G R}=I_{G} / I_{R}$ and $r_{B R}=I_{B} / I_{R}$ are constant for all pixels $(x, y)$ independent of the dot product of the normal and illumination vectors $(N(x, y) \cdot S(x, y))$ (since they cancel out). In practice, however, due to imaging artifacts, the ratios are more defuse and therefore multiple ratios may be detectable over a patch. Given a dry patch, $P_{d}$, we compute a set of $\left(r_{G R}, r_{B R}\right)$ pairs. If the patch were perfectly uniform (in terms of surface normals), a single pair will be found, but for complex surfaces there may be several such pairs. We histogram the normalized $G / R$ and $B / R$ values to compute these pairs. Let $S_{d}$ denote the set of these ratios computed over $P_{d}$.

As a result of the above parametrization, the red albedo, $A_{R}$, is unknown and it will be searched for optimal fit and $A_{G}$ and $A_{B}$ are computed from the $S_{d}$ ratios.

Mall and da Vitoria Lobo [4] proposed that assuming a rough surface, the maximum reflected brightness, $I_{\max }$, can be used as a denominator to normalize all values and generate relative albedo values. In reality, even under these assumptions, $I_{\max }$ is the lower-bound value that should be 
used as denominator to infer the albedo of the patch. Moreover, the values acquired by the camera are subject to automatic gain, white balance and other processing that tend to change numerical values. For example, a surface with albedo equal to 1, may have a value of 180 (out of $256 \mathrm{lev-}$ els), and therefore mislead the recovery of the true surface albedo (i.e., suggesting a lower albedo than 1).

The optics framework requires absolute albedo values to predict the wet albedo of the surface. Therefore, the reflectance values should be normalized with respect to an unknown $R_{\text {white }} \geq I_{\max }$ (typically) which represents the absolute value that corresponds to the intensity of a fully reflective surface under the same imaging conditions (including unknown camera imaging parameters, and a normal and illuminant dot product equal to 1.0). Note that for an ideal image acquisition an albedo of 1 corresponds to $R_{\text {white }}=256$, but in practice $R_{\text {white }}$ can be lower (e.g., for white balance) or higher than 256 (e.g., camera gain).

Determining $R_{\text {white }}$ involves a search for the best value in the range $I_{\max }$ to $I_{U \text { pperBound }}$. While $I_{\text {UpperBound }}$ can be chosen as a large number, the computational cost is prohibitive. Instead, we observe that if we assume that the patch includes all possible surface normal orientations, then the maximum intensity, $I_{\max }$ corresponds to $(N(x, y)$. $S(x, y))$ being 1.0 while minimum intensity $I_{\min }$ corresponds to $(N(x, y) \cdot S(x, y))$ near zero, for the unknown albedo $A$ (see Equation 7). Let $\vec{n}$ denote a vector of the values of all the normals multiplied by the illuminant direction (these values span the range $0 . .1$ ). Therefore, the brightness of an object with an albedo of 1 in these unknown imaging conditions (and including the camera's image processing) can be computed as

$$
I_{U \text { pperBound }}=256 * \max (A * \vec{n})+256 * \max ((1-A) * \vec{n})
$$

where 256 is the camera's intensity output range (assuming no saturation occurred). This is equal to

$$
I_{U \text { pperBound }}=I_{\max }+\left(256-I_{\min }\right)
$$

$I_{\max }$ and $I_{\min }$ may be subject to noise and imaging factors that may create outliers, so we approximate the intensity values as a gaussian distribution with a standard deviation $\sigma$ and assign $I_{\max }-I_{\min }=4 * \sigma$ cropping the tail values and capturing near $97 \%$ of the distribution, so that $I_{U \text { pperBound }}=256+4 * \sigma$. This gaussian assumption is reasonable for a rough surface but for a flat surface, $\sigma$ is near zero, and therefore we use $I_{U \text { pperBound }}=256+100$ as an arbitrary value. Note that $I_{U \text { pperBound }}$ reduces the range of the search for the best $R_{w h i t e}$ and not the quality of the results. We use the largest value of $I_{U \text { pperBound }}$ computed for each of the $R G B$ channels for all searches.

$I_{\max }$ may be subject to automatic gain amplification during acquisition. Therefore, the range of values for $R_{\text {white }}$ is expanded to be from $0.75 * I_{\max }$ to $I_{\text {UpperBound }}$.
The choice of 0.75 is arbitrary since it assumes that the gain is limited to $33 \%$ of the true values, and one could choose a different values.

Given a pixel from a dry patch, $P_{d}$, we can convert its value to a wet pixel

$$
P_{w}(x, y)=P_{d}(x, y)+\left(\left(1-a_{d}\right)-\left(1-a_{w}\right)\right) * R_{w h i t e}
$$

where $a_{w}$ is calculated using Equation 5 given a specific $a_{d}$. Equation 10 is applied to each of the $R G B$ channels using the respective parameters.

\subsection{Liquid Spectral Absorption}

The model described so far assumed that the spectral absorption of the liquid film itself is near zero across all wavelengths. This is a reasonable assumption for water since it can be treated as translucent given the negligible thickness of the liquid present at the surface. We next consider water-based liquids that have different absorption rates across wavelengths such as coffee and wine (even at negligible thickness). We assume a refractive index that is equal to water, however we assume that $q_{r}, q_{g}, q_{b}$ represent corrective absorption rates in $R G B$, respectively. These corrective rates modify the darkening due to water-based wetness. The real liquid absorption rates are computed as

$$
\begin{aligned}
& L_{r}=q_{r} \\
& L_{g}=a_{w g}-a_{w r}+q_{g} \\
& L_{b}=a_{w b}-a_{w r}+q_{b}
\end{aligned}
$$

where $a_{w r}, a_{w g}, a_{w b}$ are the respective wet surface absorptions for red, green and blue, respectively (for water). Equation 10 is modified to account for the liquid absorption rates:

$P_{w}(x, y)=P_{d}(x, y)+\left(\left(1-a_{d}\right)-\left(1-a_{w}\right)-(1-q)\right) * R_{w h i t e}$ (12)

where the respective parameters for each of the $R G B$ channels are used. Note that Equation 11 computes relative absorption rates with respect to $q_{r}$, so that we recover only the differences in absorptions between the $R G B$ channels. Nevertheless, these relative absorptions are informative and sufficient since the absolute values are intertwined with the intensity of the illuminant. For example, adding a constant absorption of 0.1 to each of $L_{r}, L_{g}, L_{b}$ is equal to decrease in reflected light equal to a $10 \%$ loss of illuminant intensity.

Absent prior information, we search the full range of possible values between $0-1.0$ for each variable. In practice, we can, in most cases, limit the search to values between $0.0-0.5$ since higher values are likely, when combined with the increased absorption due to wetting, to drive total light absorption to 1.0 which represents a black object. In cases where the $P_{w}$ shows complete absorption of a wavelength (e.g., a thick layer of wine or coffee), the $0 . .1$ range is searched. Moreover, values that represent equal absorptions, $q_{r} \approx q_{g} \approx q_{b}$ are unnecessary to consider since 
they are functionally equivalent to water (but they do contribute uniform darkening in all channels that is automatically captured in the computation of the absorption values of the material). The search is conducted in small increments of 0.02 .

\subsection{Similarity Metric}

The synthesized wet patch $P_{s}$ is scored against $P_{w}$. A useful similarity metric is the well-known Earth Mover's Distance [7] (EMD). The distance is computed between the size-normalized histograms of the two patches. The smaller the distance, the closer the appearance between the synthesized and true wet patches. Given that these patches are typically taken from different parts of the same image, we assume that the dry and wet patches are of the same material as well as have similar surface normal distributions. If the distributions of surface normals between the two patches violate this assumption, we have a suboptimal similarity metric. Devising a metric that accounts for different and unknown distributions of surface normal remains an open problem. Note that EMD is not suitable for comparing different materials (e.g., if the wet and dry material are of two different wood species).

\section{Search Space}

We summarize the search parameters to determine the best synthesis, $P_{s}$, of $P_{d}$ given $P_{w}$. The refractive index of the material, $n_{r}$ is unknown. Refractive indices of materials vary widely, with air being near 1.0 and the highest measured material (a synthetic material) is 38.6. Common materials, however, tend to fall between $1-5.0$. As a result, we perform a search on all values of $n_{r}$ between $1.1-5.0$ in increments of 0.1 (note that if we assume the material to have higher refractive index than water, the search can be made between $1.5-5.0$ ). Note that $n_{r}$ is dependent on light wavelengths (i.e., light wavelengths have slightly different speeds in the same medium), but accounting for this variation in the search process is computationally expensive. Therefore, we use the same $n_{r}$ for the three channels.

We assume the liquid to be water-like, so that $n_{l}$ is known. Specifically, we assume that $n_{l}=1.331$ for the red channel, $n_{l}=1.336$ for the green channel, and $n_{l}=1.343$ for the blue channel. This assumption is suitable for most water-based liquids such as coffee, wine, etc. (in practice, the ethanol in wine increases the refractive index slightly, and coffee particles increase it upto 1.5). Other liquids, such as oil, have different refractive indices, but since we assume no prior information, we employ the water refractive indices even when oil may be involved.

The absorption rate of the dry material, $a_{d}$, is unknown and falls in the range $0-1.0$. The discussion in subsection 3.2 uses the albedo $A_{R}$ as a variable and derives the green and blue albedo values, and thus their absorptions accord- ingly. Therefore, we perform a search over all values between $0.05-0.95$ in 0.05 increments for $a_{d_{R}}$. The values $I_{\min }, I_{\max }$ and $I_{U p p e r B o u n d}$ are pre-computed and then a search for optimal $R_{w h i t e}$ is computed in increments of 20 units for the range $0.75 * I_{\max }$ and $I_{U \text { pper Bound }}$.

Depending on the expected liquid, we can limit the search to water, or search in a reduced $3 \mathrm{D}$ space of liquid correction absorption rates, $q_{r}, q_{g}, q_{b}$, as discussed in section 3.3. Algorithm 1, below, is for the case of water, but can be adjusted for an unknown liquid.

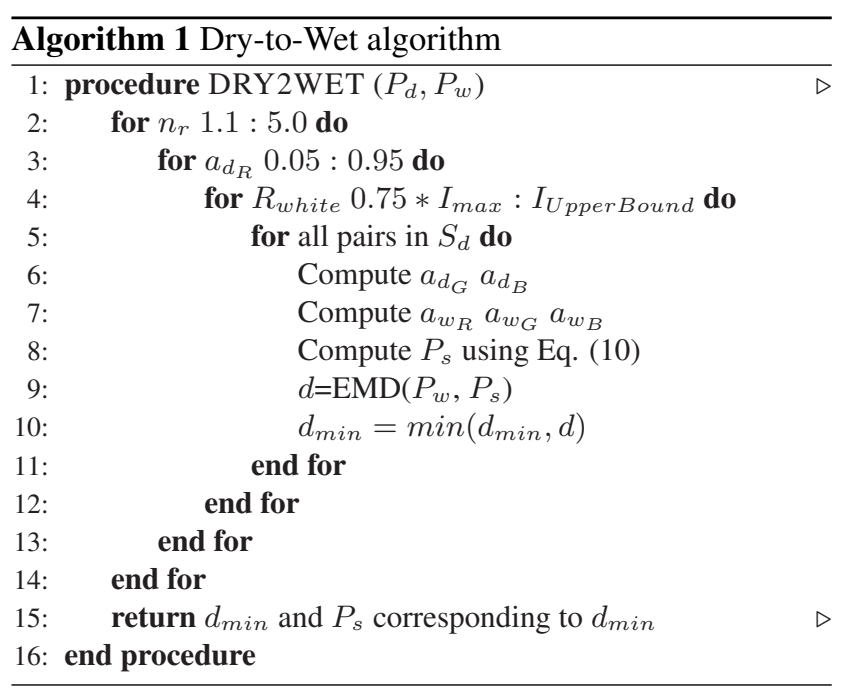

\section{Experiments}

We conducted experiments on three data sets: collected by us, collected from the web, and a controlled set of drying objects collected and described in $\mathrm{Gu}$ et al. [6]. The experiments answer the question: given a dry patch, $P_{d}$ and a patch likely to be wet $P_{w}$, what are the best parameters that make $P_{d}$ look most similar to $P_{w}$ ? The answer allows uncovering physical information about the liquid and the material which is valuable for computer vision. The answer may also indicate that no wetting process can make $P_{d}$ look like $P_{w}$, which is also valuable since it suggests that the two patches differ in more significant ways.

Note that we focus on applying a physically-motivated model to the problem and not an image-based appearance transformation. One could pose the problem differently by computing a transformation (that has nothing to do with wetting) that maximizes the similarity between a transformed $P_{d}$ and $P_{w}$. But such transformation does not uncover information about the physical process that is involved and is ultimately less insightful.

The patches $P_{d}$ and $P_{w}$ are manually delineated. The border area between the patches is neither fully dry or wet. Therefore, the border area is rarely synthesized properly. We exclude these boundary pixels from EMD computation between $P_{s}$ and $P_{w}$. 
Empirically, we observed that EMD distances below 20 indicate close resemblance and below 10 are near identical images. Note that EMD does not capture the spatial color variations (i.e., texture differences). In all figures below, the numeric values show the EMD distance, followed by $\left(n_{r}, R_{w h i t e}\right)$, the next row shows the respective albedo values $A_{R}, A_{G}, A_{B}$. In the images of the colored liquids, the third row shows the albedo of the liquid $A L_{R}, A L_{G}, A L_{B}$.

Figure 3 shows the results of the closest synthetic wetting of a dry material (images taken from [6]). These images were taken under controlled illumination but at different times, as the initially wet material dried. The top row shows the dry materials, the middle row shows the real wet material, both are provided by [6]. The bottom row of images shows the computed wet materials using our algorithm. Below each image we provide the physical parameters that our algorithm uncovered, assuming the liquid is water. Note that most of the true wet images have some specular reflections that are not generated by our model. The materials are (left to right), rock, wood, cloth, wood, felt, paper, cardboard, brick, wood, cloth, cloth and granite. The results indicate that wood is the least successfully analyzed material. The wet wood has increased spectral divergence in colors beyond what the dry material exhibits and therefore does not appear to be correctly captured by the model. Specifically, the wet wood appears to absorb more of the blue and green light relative to red, and therefore the wood is tinted brown-red. We discuss this issue further in Section 6.

Figure 4 shows images we acquired of different wet materials. From left to right all images have a darker wet patch: yellow paper (wet on the right side), paper towel, large area of a cap, a smaller part of the same cap, blue paper, orange fleece material, grey/blue paper, green paper, orange fabric, and grey/blue fabric. The distances are largest for the complete green cap and blue paper. The reason is that the surface normal distributions vary between the wet and dry patches, and therefore the EMD is not a suitable metric (see discussion in subsection 3.4). The smaller part of the cap shows very good synthesis of the dry patch.

Figure 5 shows a collection of images of water-based wetting of different materials downloaded from the web. From left to right, raster scan, partially wet: two cardboard images, concrete, yellow brick, three types of wood, blue fabric, two images of different types of sand, red tile, red brick, blue/green brick, striped shirt and grey pants. Two of the wood images show the largest distances and a discussion of likely reasons is provided in Section 6. The rest of images are close to the real wet areas in each image ignoring the borders between patches.

Figure 5 shows a collection of images downloaded from the web of non-water wetting. From left to right, raster scan, partially wet: coffee on carpet, coffee on wood, wine on carpet, olive oil on humus, olive oil on wood, tea on fabric, coffee on fabric, two images of coffee on carpet, wine on tile, wine on carpet, wine on granite, same image but applying a water model, wine on carpet, coffee on plastic table cloth, coffee on carpet, coffee on shirt, same image but applying a water model, wine on yellow napkin, and soy sauce on yellow napkin (the last two images are acquired by us). The liquid color is rendered with intensity that is close to the wet area. The wine on granite and coffee on shirt are used to also demonstrate the results of the water model as opposed to accounting for different spectral absorptions. Overall the distances are low with exception to the olive oil on wood and wine on white carpet (middle of the bottom group). The olive oil on wood maybe related to explanations in Section 6 while the wine on carpet shows marked difference in surface normals between the dry and wet patches (the wet patches are in focus while the dry patch is blurred).

\section{Open Challenges}

The experiments indicated that in some images of wet wood, the model is not accurate. Figure 7 shows an image of an outdoor deck, a part of a wetted area used for an experiment, and the synthesized dry patch using our model. The dry wood appears nearly perfectly grey, while the wet wood is brown. The wet pixels show high absorption of green relative to red, and even higher absorption of blue relative to green and red. The model does not predict this result given that the liquid is water. A similar phenomenon was observed in some experiments in Figures 3 and 5.

We suggest two conjectures as to why this occurs. The first has to do with image acquisition, and suggests that perhaps the camera is overstating the amount of blue and green light reflected at the dry patch. The second is that these woods and their resultant images have a more complex wetting process. Specifically, it is possible that this wood is composed of 2 layers, the first is very thin and tends to have only a hint of the spectral properties of the wood, and the second layer reflects the full spectral attributes of the wood. The top layer may come to exist due to environmental degradation or dust, but may not exist in freshly cut wood. For the dry wood in Figure 7 the reflectance is mostly the result of reflection from the top layer, while upon wetting, the second layer is reached by the water and thus it becomes the dominant source of reflectance. Unfortunately it remains an open challenge to explain these deviations from the model.

Differences in the distributions of the surface normals between the dry and wet patches make it harder to determine similarity (even if a different metric than EMD is used). This is general computer vision problem that is not specific to wetting, but is made more challenging by the complexity of the wetting process. 

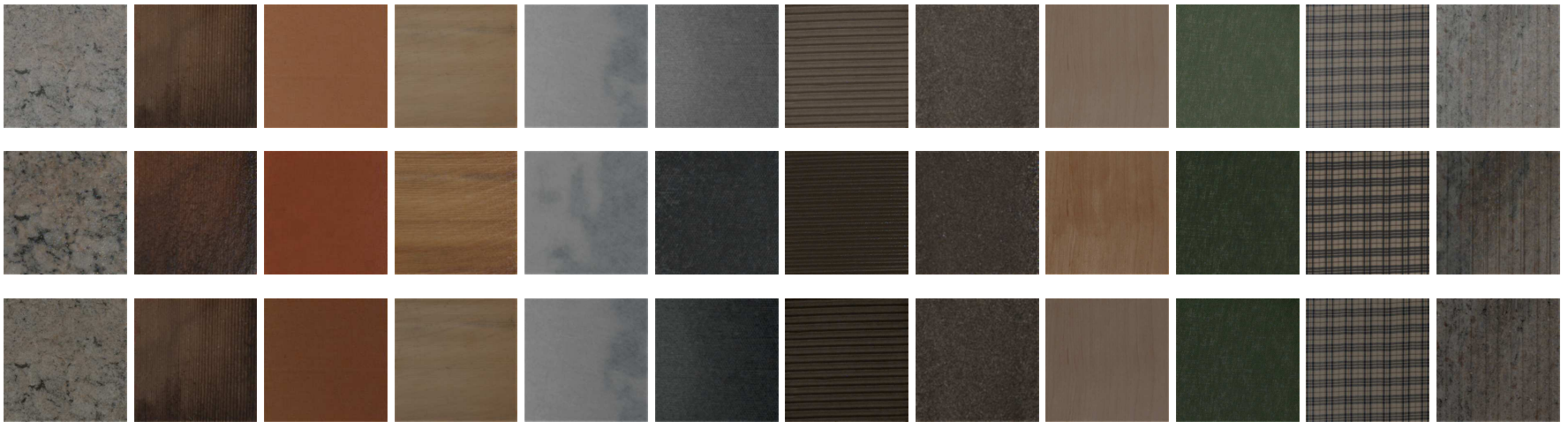

$\begin{array}{lr}16.4(5.0,162) & 9.2(5.0,247) \\ (0.60,0.61,0.62) & (0.15,0.14,0.12)\end{array}$

$\begin{array}{ll}3.0(5.0,154) & 24.1(5.0,146) \\ (0.10,0.09,0.09) & (0.10,0.09,0.08)\end{array}$

$1.5(4.8 .121)$

$\begin{array}{rr}13.3(2.7,131) & 7.0(3.8,157) \\ (0.15,0.15,0.15) & (0.30,0.29,0.28)\end{array}$

Figure 3. Top row, images of dry material, middle row, images of wet materials (water), and bottom row the synthesized wet images.
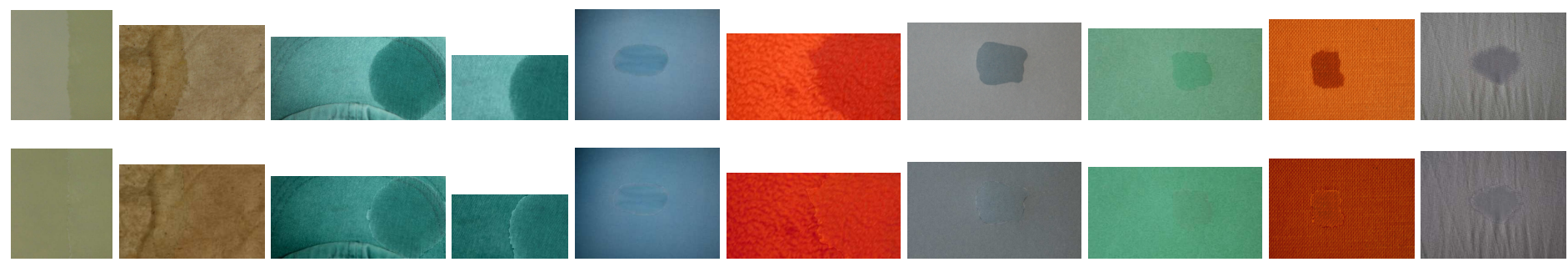

$1.2(3.1,122)$
$(0.90,0.91,0.77)$

$31.95(3.0,173)$

$3.4(4.9,244)$

$29.4(2.1 .138)$

$12.06(2.3,191)$

$8.6(3.1214)$

$9.9(2.8 .159)$

$10.2(2.9,191)$

$19.7(1.8,158)$

Figure 4. Top row, input images with wet patches. Bottom row, dry patches synthesized into wet patches assuming water. From left to right, yellow paper, brown paper towel, large area over a cap, small area of the cap, blue paper, orange fleece, grey/blue paper, green paper, orange fabric and grey/blue fabric.

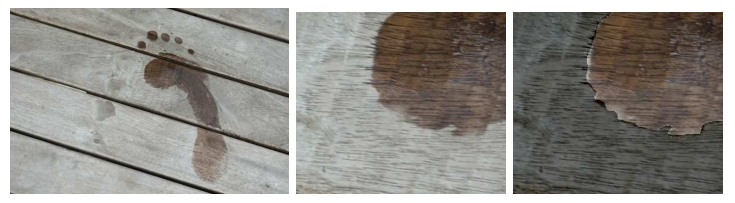

Figure 7. Left to right, footprints on dry deck, input for our algorithm, and synthesized output.

\section{Summary}

In this paper we investigated the problem of visual appearance change as liquids and rough surfaces interact. The problem assumes that two patches, the first is known to be dry and the second is possibly wet are given. Liquid attributes that are close to water, but also allow for varying absorption rates across spectral wavelengths allow accounting for unknown liquids suchs as coffee, wine and oil. Our experiments indicate an ability to explain wetting effects in different materials and under unknown imaging conditions.

\section{References}

[1] A. Angstrom. The Albedo of Various Surfaces of Ground, Geographic Annals, vol. 7, 1925, 323-342.

[2] T. Teshima, H. Saito, M. Shimizu, and A. Taguchi. Classification of Wet/Dry Area Based on the Mahalanobis Distance of Feature from Time Space Image
Analysis. IAPR Conference on Machine Vision Applications, 2009, 467-470.

[3] J Lekner and M. C. Dorf. Why some things are darker when wet, Applied Optics, (27)7, 1988, 1278-1280.

[4] H. Mall and N. da Vitoria Lobo. Determining Wet Surfaces from Dry. ICCV, Boston, 1995 , 963 - 968.

[5] H. Jensen, J. Legakis, J. Dorsey. Rendering of Wet Materials. Rendering Techniques 99. Eds. D. Lischinski and G. Larson. Springer-Verlag, 1999, 273-282.

[6] J. Gu, C. Tu, R. Ramamoorthi, P. Belhumeur, W. Matusik and S. K. Nayar. Time-varying Surface Appearance: Acquisition, Modeling, and Rendering. ACM Trans. on Graphics (also Proc. of ACM SIGGRAPH), Jul, 2006, (25)3,762 - 771.

[7] Y. Rubner, C. Tomasi, L. J. Guibas. A Metric for Distributions with Applications to Image Databases. Proceedings ICCV, 1998: 59-66. 

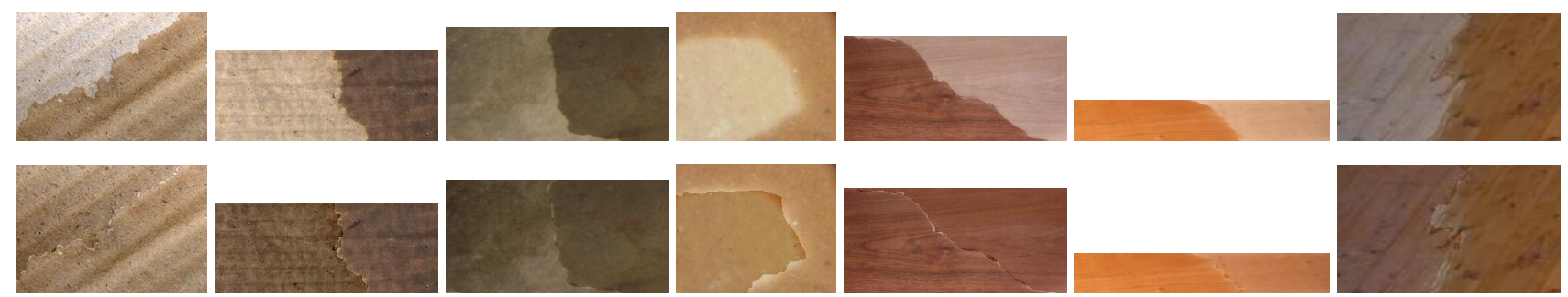

$7.6_{(0.85,351)}^{(3.0 .80,0.72)}$

$25.7(5.0 .290))$

$15.5(4.7 .7131)$

$8.9(4.1,250)$

$13.0(2.5,270)$

$48.5(3.2,173)$

$39.0(2.1,189$
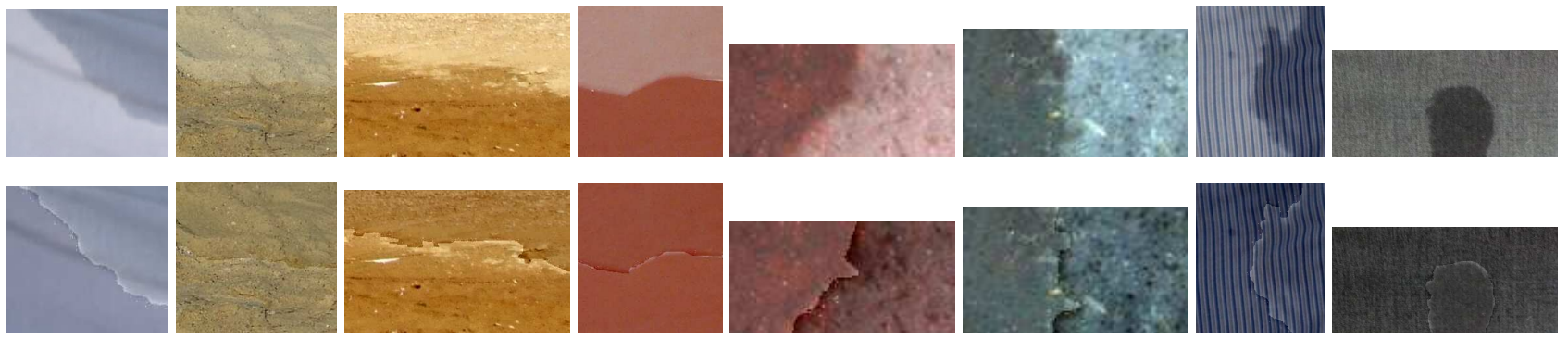

$7.2_{(0.70,0.71,0.78)}^{(2.6219)}$

$11.1_{(0.80,0.157,0.60)}^{(1.8 .177)}$

$29.7(5.0,291)$
$(0.75,0.63,0.47)$

$4.8(3.1,263)$
$(0.90,0.71,0.62)$

$21.1_{(0.55,0.0 .2917)}$

$28.2(5.0 .331)$

$3.4(5.0 .220)$
$(0.55,0.56,0.61)$

$13.5(3.9 .245)$
$(0.20,0.21,0.20)$

Figure 5. Web images, top row is input, and second row is synthetic wetting.

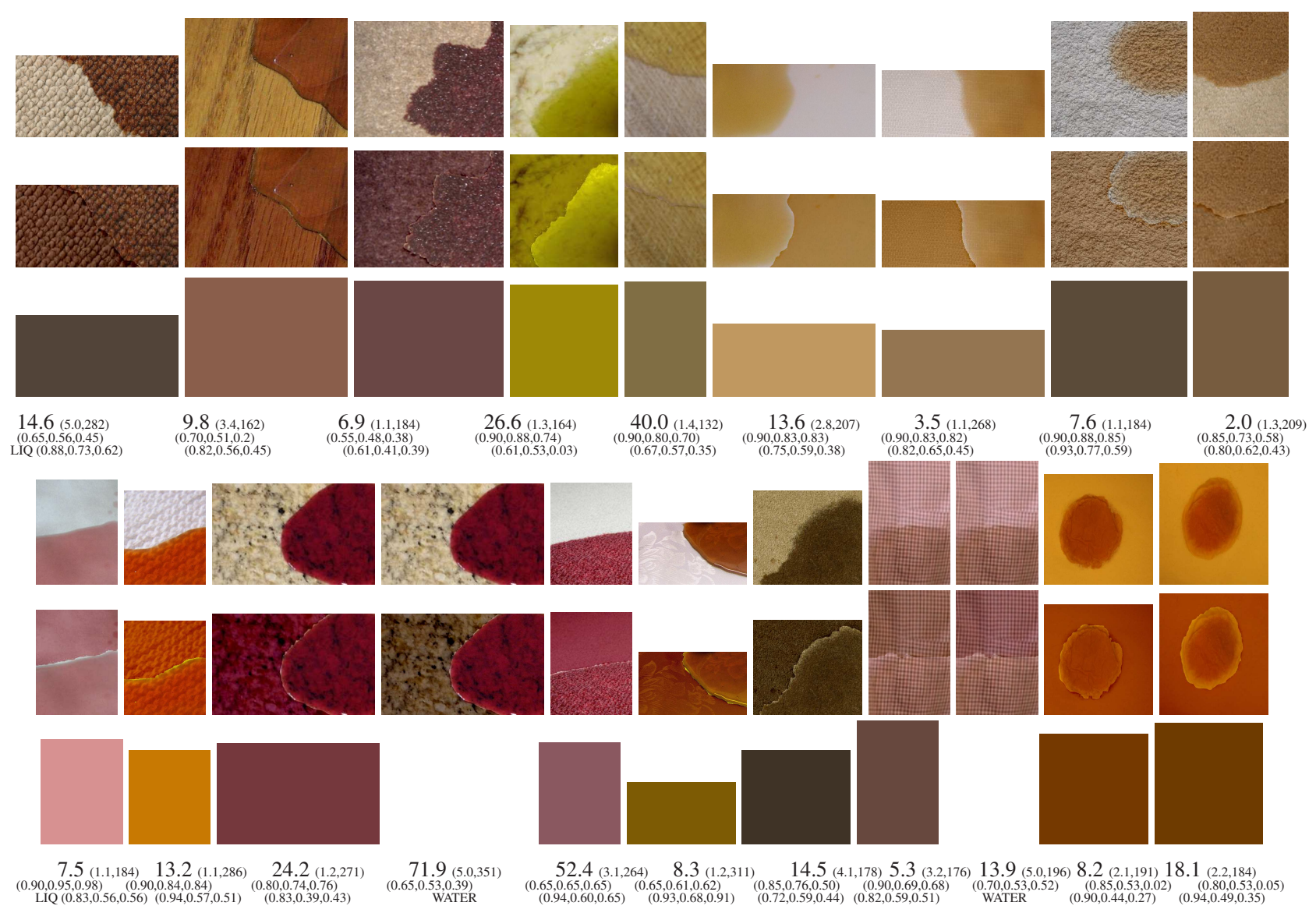

Figure 6. Web images, top to bottom rows: input, synthetic wetting, and liquid albedo. 